\title{
Correlation method for pattern recognition
}

\author{
P.V. Yezhov, A.V. Kuzmenko, V.A. Komarov \\ International Center "Institute of Applied Optics", NAS of Ukraine, 10G Kudryavskaya, 04053 Kyiv, Ukraine \\ E-mail:yezhov@iop.kiev.ua
}

\begin{abstract}
A new approach towards solving the pattern recognition problems in hybrid optical/digital correlators is suggested. The method is based on the authors' know-how-principle of transforming the field intensity or amplitude data on the identified object assuming that these data may be entered into a computer by ordinary methods. This principle allows to solve the unification of the correlation functions and recognition attributes selection problem using a unified, identified object's class-independent method. Presented experimental results confirm applicability of this approach.
\end{abstract}

Keywords: pattern recognition, optical-digital correlators

Paper received 04.11.02; accepted for publication 17.12.02.

\section{Introduction}

It is known that, the recognition criteria for objects exposed to identification procedure in hybrid optical-digital correlators [1-2] are not universal, i.e. the criteria, suitable for one class of objects are unsuitable for objects of other classes. In addition, object classification in optics is rather arbitrary, and the recognition criteria are empirical. Also complicating the problem is the fact that correlation functions, obtained in the known recognition schemes, in the majority of cases are complicated and their unambiguous interpretation (acknowledgment or rejection of the object recognition) is hindered.

Considering the above, the authors suggested a method for processing the input images of the objects [3] in hybrid optical-digital correlators, the essential feature of which is that each object is assigned with unique twodimensional phase distribution, calculated by a computer. One such distribution supersedes (substitutes) the object at the stage of making the holographic filter archive, and the second supersedes (substitutes) the object at the stage of recognition (it is used instead of the object).

The key point is as follows: the object and its corresponding phase distribution, have no point-wise or frag- ment-wise coincidence; the phase distribution belongs to a set of random phase distribution having Fourier-spectrum uniform by amplitude and producing $\delta$-like autocorrelation signal.

\section{Experiment and results}

The method was approved in a hybrid optical-digital correlator shown in Fig. 1. Amplitude 64×64 element objects, with fragments ordered to different extents were used.

The recognition procedure consists of the following steps:

1) the reference object image (1) is fed to the CCD camera (2) of the input data system;

2) the phase distribution corresponding to the reference object is computed (3);

3 ) the object to be recognized (1) is fed to the CCD camera (2);

4) the phase distribution corresponding to the object under recognition, is computed (3);

5 ) the correlation integral of two phase distributions corresponding to the objects, is calculated (3).

Using the described procedure, we performed the modeling experiments with various types of half-tone and 


\section{P.V. Yezhov et al.: Correlation method ...}

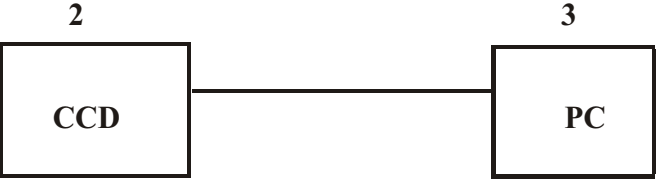

Fig. 1. Scheme of optical-digital correlator. 1 - object plane; 2 - input data system; 3 - computer.
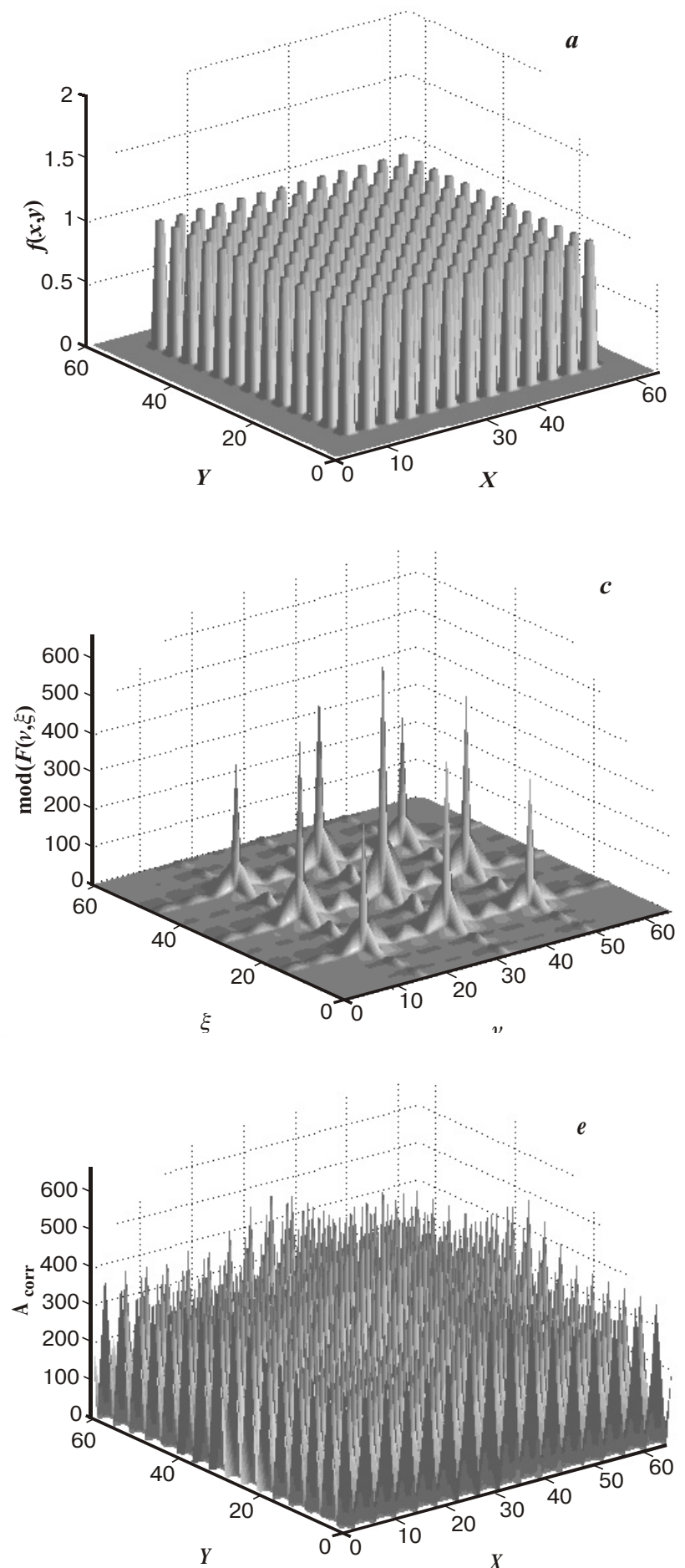

binary objects. Figs $2 \mathrm{~A}$ and $2 \mathrm{C}$ show an example of an object with regular structure and spectrum thereof, for which the recognition was performed. Figs $2 \mathrm{~B}$ and 2D show phase distribution corresponding to the object (Fig. 2A) and Fourier spectrum thereof. Figs $2 \mathrm{E}$ and $2 \mathrm{~F}$ show the correlation signals received using standard and sug-
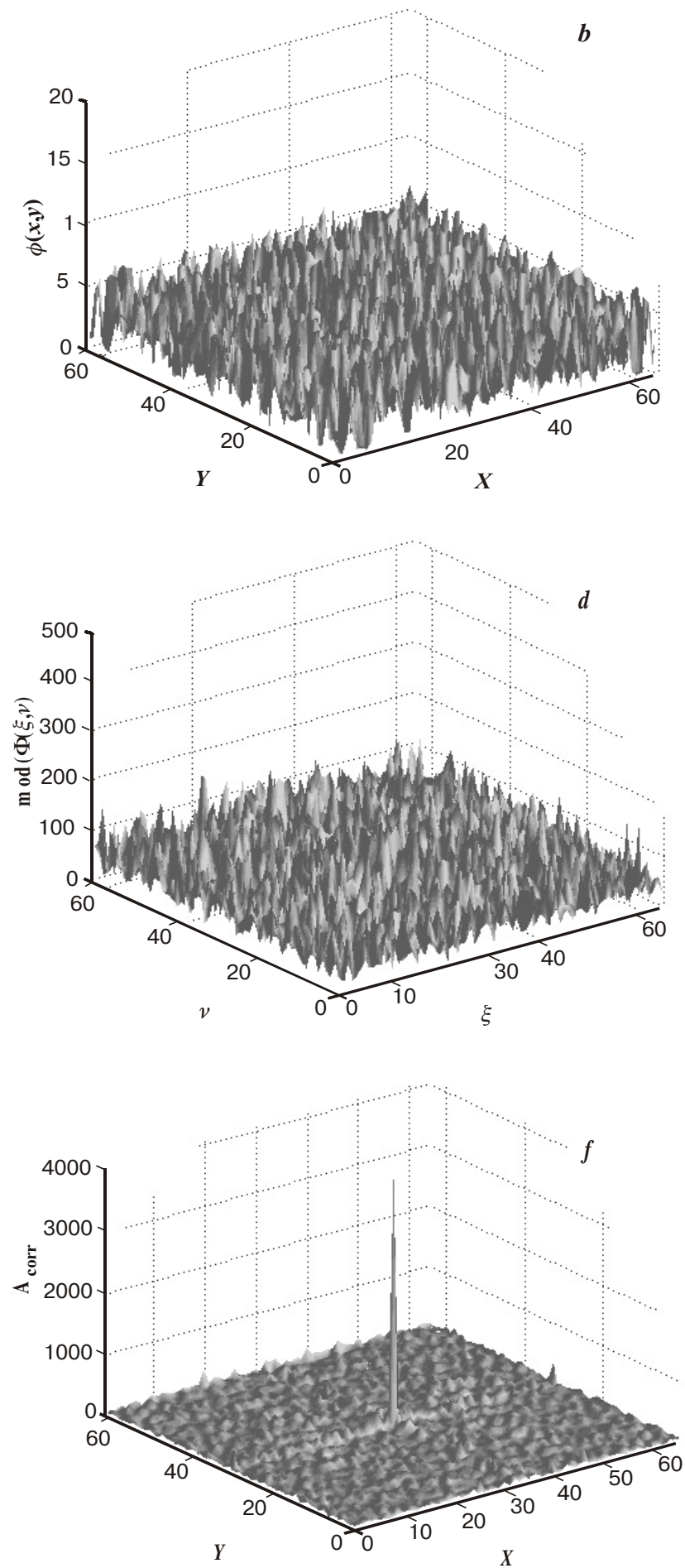

Fig. 2. Illustration of the recognition methods. 1) object with regular structure (A) and Fourier-spectrum thereof (C), for which the recognition was performed; the correlation signal (E) corresponding to the object; 2) phase distribution (B) corresponding to the object (Fig. 2A) and Fourier spectrum thereof (D); the correlation signal (F) corresponding to the object (B). 


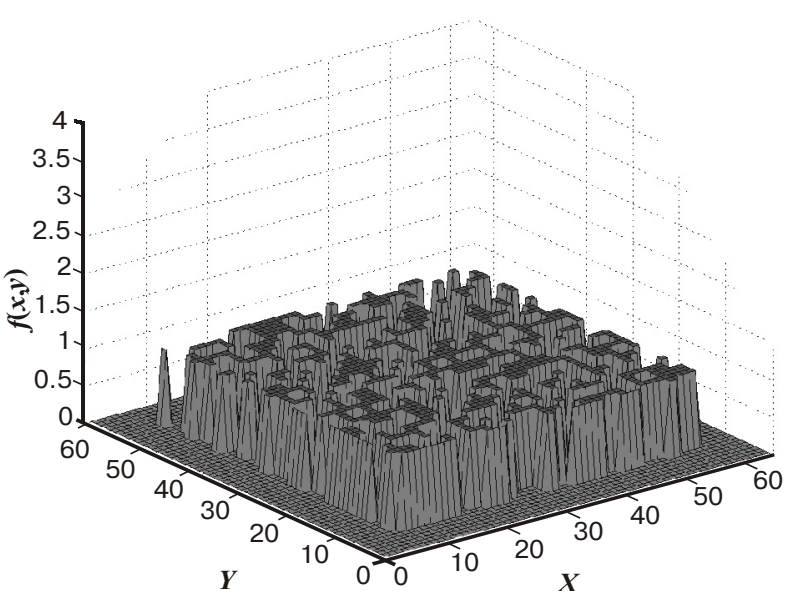

Fig. 3. Object with random distribution of elements.

gested recognition methods. It should be mentioned, that autocorrelation function form for random phase distribution corresponding to various objects is unified, i.e. object type-independent. The signal/noise ratio for autocorrelation in our experiment was $36 \mathrm{~dB}$ (Fig. 2F). This value is typical at handling other objects and depends only on the number of the allowed object elements.

Fig. 3 shows an object with random distribution of elements, for which the recognition was performed in case of its distortion by irising. Fig. 4 shows a dependency of the correlation signal amplitude upon the object degree of irising. Curve 1 corresponds to the suggested method, curve 2 belongs to the classic recognition method, $\left(1-S / S_{0}\right)$ is a parameter, defining the degree of irising, $S_{0}, S$ are the areas of the objects before and after irising.

As a universal recognition criteria, it is convenient to select the dispersion (relative to average) of phase distribution Fourier spectrum amplitude. In addition, this parameter allows to evaluate homogeneity of their Fourier spectra, that is important at filter registration on holographic medium, and to control the phase distribution computation process.

Therefore, this method essentially increases the recognition certainty. It should be mentioned that the sensi-

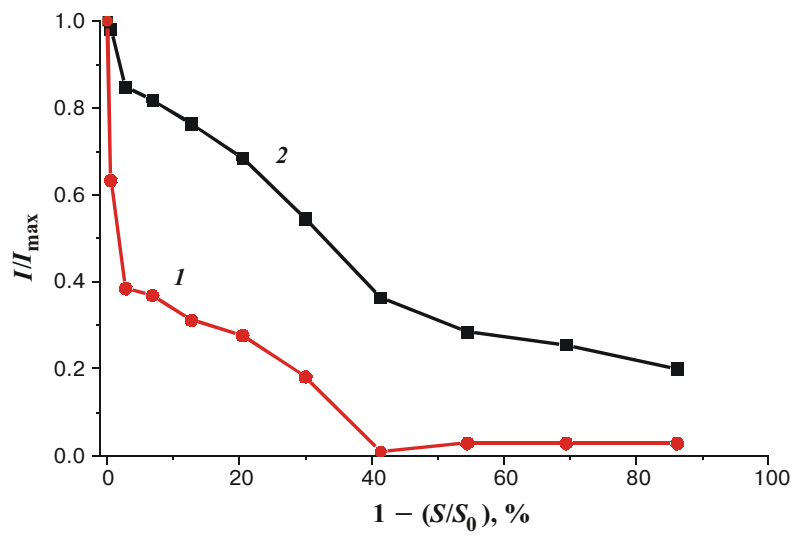

Fig. 4. Dependence of the correlation signal amplitude upon the object's (Fig. 3) degree of irising. 1) curve corresponds to the suggested method; 2) curve belongs to the classic recognition method.

tivity of the method to any other distortion types in the identified objects may be adjusted at the stage of phase distribution computation, that is different from classic methods, where it remains constant.

The described method may be implemented in the standard optical-digital VanderLugt and joint Fouriertransform correlator types.

\section{Conclusion}

The universality of the method consists of unification of the correlation functions for the identified objects and unified approach to the recognition attributes selection.

\section{References}

1. Applications of Optical Fourier Transform // Edited by Henry Stark, London, “Academic Press",1982.P.386.

2. L.I. Muravsky. Phase image processing in the optics and optical-digital correlation systems. Ternopil.: "Serpen”,1999. P.187.

3. A.V. Kuzmenko, V.A. Komarov P.V. Yezhov, Method of phase determination by means of the amplitude data. Patent of Ukraine № 48880A. Bulletin №8 2002. 